\title{
Téoros
}

Revue de recherche en tourisme

\section{Modèle de coopération entre Parcs Canada et le milieu universitaire dans les parcs nationaux canadiens : cas de l'Université de Sherbrooke}

\author{
Jean-Marie M. Dubois, André Poulin et Léo Provencher
}

Volume 14, numéro 1, printemps 1995

Les parcs : des réseaux en mutation

URI : https://id.erudit.org/iderudit/1077057ar

DOI : https://doi.org/10.7202/1077057ar

Aller au sommaire du numéro

Éditeur(s)

Université du Québec à Montréal

ISSN

0712-8657 (imprimé)

1923-2705 (numérique)

Découvrir la revue

Citer cet article

Dubois, J.-M. M., Poulin, A. \& Provencher, L. (1995). Modèle de coopération entre Parcs Canada et le milieu universitaire dans les parcs nationaux canadiens : cas de l'Université de Sherbrooke. Téoros, 14(1), 46-49. https://doi.org/10.7202/1077057ar 


\title{
Modèle de coopération entre Parcs Canada et le milieu universitaire dans les parcs nationaux canadiens : cas de I'Université de Sherbrooke
}

\author{
Jean-Marie M. Dubois, André Poulin, Léo Provencher*
}

\section{$+$}

Dans l'esprit de la majorité des citoyens, les parcs sont des espaces pour se recréer ou pour admirer la nature. La plupart des visiteurs les utilisent pour se familiariser avec le milieu régional puisque les pares en sont normalement le reflet; de là, ils essaiment vers d'autres attractions. Afin de pouvoir assumer pleinement leur rôle touristique, les parcs doiventsavoir éduquer la population et lui présenter une information intéressante et captivante. La vocation éducative des parcs, qui fait partie intrinsèque de leur mission, est surtout connue à travers les programmes d'interprétation qui sont offerts aux visiteurs et aux groupes scolaires des niveaux primaire etsecondaire. Cette vocation éducative est cependant beaucoup moins manifeste aux niveaux collégial et universitaire où, dans la plupart des institutions, l'éducation à la recherche est moins publicisée ou mêeme inexistante dans le cas des parcs. A cet égard, Parcs Canada se démarque par une politique en matière de recherche et c'est cet aspect de la vocation des parcs qui fera l'objet du présent article. Il sera question du partenariat développé avec l'Université de Sherbrooke et de sa concrétisation particulière au Parc national Kouchibouguac, au Nouveau-Brunswick.

\section{Politique générale de Parcs Canada}

Parcs Canada s'est donné une première politique en matière de conservation de territoires représentatifs des milieux naturels canadiens peu après la constitution du premier parc, soit celui de Banff en 1885 (Patrimoine canadien, 1994). L'avantdernière, promulguée en 1979 , s'inspire, entre autres, de la Comention concernant la protection du patrimoine culturel et naturel mondial, ratifiée en 1972 et dans laquelle le Canada s'est engagéà identifier et à délimiter des sites d'intérêtmondial. La dernière,

\footnotetext{
- Messieurs Jean-Marie M. Dubois et André Pouln sont professeurs au département de gégraphie et télédétection de I'Université de Sherbrooke. Monsieur Leo Provencher est chargé de cours at
} ce mëme département. qui date de 1994, s'inspire de la Convention sur la diversité biologique, adoptée à Rio de Janeiro en 1992, ainsi que du Plan de Caracas de 1991 par l'International Union for Conservation of Nature. À cet égard, «Parcs Canada encourage la protection des écosystèmes et habitats naturels, le maintient et le rétablissement de populations indigènes viables de différentes espèces dans leur environnement naturel, et la bonne gestion environnementale des aires environnantes ou voisines» (Patrimoine canadien, 1994, p. 9). Il en découle que, dans les parcs nationaux, on peut étudier les milieux naturels tout aussi bien que faire l'interprétation de la façon dont l'environnement a influé sur l'évolution sociale du pays et ce, en plus de pratiquer une grande variété d'activités de plein air (Environnement Canada, 1990a). De plus, on y introduit la vision qu'un parc n'est pas un enclos fermé, mais qu'il fait partie d'un «touto avec les écosystèmes environnants, «tout» qu'il faut aussi connaitre. En effet, ceci veut dire que, pour chaque parc ou lieu historique, il faut, entre autres, évaluer l'état des ressources patrimoniales et l'état deleur miseenvaleur. Il faut aussiproduire l'inventaire de ce qui menace ou peut menacer ces ressources de l'intérieur ou de l'extérieur ainsi que ce qu'il est possible de faire pour préserver l'intégralité écologique des écosystèmes qu'on y trouve (Environnement Canada, 1990b).

Pour arriver à leurs fins, les parcs doivent établir des plans de gestion révisés tous les cinq ans dans lesquels ils établissent des stratégies de protection des écosystèmes tant à l'intérieur qu'à l'extérieur de leurs limites. Ils doivent aussi promouvoir les travaux de recherche qui contribuent à la protection des écosystèmes et à leur compréhension par le public afin de mieux promouvoir les programmes d'interprétation.

La protection de seulement $6,9 \%$ du territoire canadien est présentement assurée par les différents paliers administratifs gouvernementaux ou par des organismes privés de conservation. Les parcs nationaux y contribuent pour $1,8 \%$, soit pour plus du quart avec 34 parcs, 7 autres prévus et 112 lieux historiques: ils sont donc des partenaires privilégiés pour les universités (Gouvernement du Canada, 1990). Au Québec, on retrouve ainsi 26 lieux historiques nationaux et les parcs de la Mauricie, de Forillon, en plus de la Réserve deparc de l'Archipel-de-Mingan et du parc marin du Saguenay. D'autres projets de parcs ont été mis de l'avant, par exemple celui du golfe Guillaume-Delisle à labaied'Hudson. Sur les 39 régions naturelles terrestres définies par Parcs Canada, 13 sont présentes au Québec, et sur les 29 régions naturelles marines, 8 y sont présentes (Environnement Canada, 1990a). La représentativité des milieux naturels québécois au sein des espaces protégés par Parcs Canada est loin d'être atteinte et beaucoup de travail reste à faire, tant aux niveaux politique que scientifique.

La politique de $1994 \mathrm{~s}$ 'articule donc autour du rôle de leadership de Parcs Canada, mais aussi *dans l'effort commun avec tous les Canadiens pour préserver ces aires. Le publicsera de plus en plus appeléà partager cetteresponsabilités (Patrimoinecanadien, 1994, p. 13). Deux des dix principes directeurs de la politique portent sur cette participation du public ainsi que sur la collaboration et la coopération. En effet, l'avis, la consultation, la participation et l'expertise d'individus, de groupes sans but lucratifoud'organismes gouvernementaux sont de plus en plus encouragés Danslecas des individus qui veulent intervenir, on passe par une politique particulière d'ententes bénévoles. Dans le cas des institutions d'enseignement supérieur, on passe par les ententes de recherche, dont le contenu est balisé par un des principes directeurs de la politique, soit celui sur les recherches et la science (Patrimoine canadien, 1994).

\section{Protocoles d'ententes de recherche avec Parcs Canada}

Le programme d'ententes bénévoles est une des bases de réalisation du protocole d'ententede recherche avecles institutions 
d'enseignement supérieurcanadiennes. En effet, chaque projet défini à partir de ces protocoles fait l'objet d'une entente bénévole avec un étudiant ou un enseignant. Habituellement, comme c'est le cas d'ailleurs avec l'Université de Sherbrooke, les projets sont définis dans le volet de service de conservation des ressources et plus spécifiquement de gestion des ressources.

Mềme s'il existe divers programmes de collaboration depuis 1966(Vallières, 1991), le programme d'entente de recherche proprement dit a été établi en 1983 (Environnement Canada, 1990b), et touche, à ce jour, environ 16 universités et collèges. Au Québec, l'Université de Sherbrooke est la seule à profiter de ce programme, saufen ce qui a trait au programme de stages coopératifs dans lequel est aussi engagée l'Université Laval. Le but poursuivi par les protocoles en vigueur est de promouvoir la recherche sur les technologies utilisables pour la gestion des parcs, ainsi que la formation relative à la planification et à la gestion des ressources naturelles dans les parcs. A cette fin, il est surtout prévu que Parcs Canada et l'institution d'enseignement puissententreprendreconjointement des projets de recherche permettant le transfert de technologies et de connaissances nouvelles entre les deuxorganismes. Le protocole prévoit aussi des échanges de personnel ou des stages de courte durée ainsi que l'évaluation réciproque des travaux danslesdomaines d'intérêtcommun. Dans ce cadre, chaque année, un comité paritaire d'orientation se réunit au moins une fois, évalue lesprojets poursuivis dans la dernière année et définit les projets à entreprendre dans l'année qui suit.

En ce qui a trait à l'Université de Sherbrooke, la Direction des ressources naturelles de Parcs Canada voulait formaliser des liens déjà établis depuis 1979, sur une base personnelle par quelques professeurs, surtout en matière de télédétection etdecartographie parordinateur. En 1986, Parcs Canada décide de financer des activitésderecherchedanslecadre demémoires de maîtrise et de contrats. Un premier protocole d'entente de recherche, établit en 1988, formalise encore plus ce lien qui se poursuit encore aujourd'hui par d'autres protocoles. Les projets se sont diversifiés pour inclure des activités d'enseignement de niveaux de ler et 3 ième cycle en plus des stages en système coopératif. Les thématiquesderecherchesont maintenant plus larges que seulement la télédétection et la cartographie, mais les volets reliés à la récréation et au tourisme sont peu développés.

\section{Protocoles d'entente de recherche avec les bureaux régionaux de PARCS CANADA}

Les bureaux régionaux de Pares Canada peuvent eux-mêmes établir des protocoles d'entente avec des institutions d'enseignement, mais ces protocoles portent habituellement sur des projets précis et ponctuels. Par exemple, en 1991 , date de l'inventairele plus récent, la région du Québec avait établi huit protocoles formels avec les universités Laval, de Montréal, de Sherbrooke et du Québec à Chicoutimi, ainsi qu'avec deux collèges, surtout pour des stages ou de la collaboration; de plus, deux contrats importants avec les universités Laval et du Québec à Chicoutimi finançaient des thèses (Vallières, 1991, pp. 18-19).

Cependant, le seul protocole d'entente de recherche connu pour l'ensemble des recherches envisageables dans une région est celui de l'Université de Sherbrooke établi, en 1992, pour cinq ans avec la région de l'Atlantique. Ce protocole formalise le travail déjà effectué dans la région, au Parc national Kouchibouguac, et encourage l'Université à s'impliquer dans d'autres parcsde cette région. Il poursuit lesmêmes objectifs que celui établi avec la Direction des ressources naturelles, à Ottawa, sauf qu'il englobe les pares historiques et les autres secteurs protégés, et qu'il encourage la recherche fondamentale, laquelle n'est pas directement liée à la gestion des parcs.

\section{Une première canadienne: un comité consultatif scientifique d'un parc national}

La mise sur pied, en 1993, d'un comité scientifique au Parc national Kouchibouguac constitue une première au Canada. Ceprojet-pilote, opérationnalisé en 1994 , regroupe à part égale, des géographes de l'Université de Sherbrooke et des biologistes de l'Université de Moncton. Il est coordonné par l'écologiste de la section de la conservation des ressources naturelles du Parc.

L'objectif principal du comité est de fournir au Directeur du parc, sur demande de ce dernier, un avis sur les activités ou les études scientifiques qui se déroulent dans son parc ainsi que sur ses plans de gestion du parc, en ce qui a trait à la conservation des écosystèmes, et à la gestion des ressources ainsi que des services. Le comité peut aussi donner son avis sur le contenu scientifique des méthodes prévues lors de l'émission d'instructions de travail ou de devis de performance en relation avec des appels d'offres ou des propositions spontanées. II peut également proposer des programmes ou des études dans des domaines d'intérêt commun au parc et aux universités. Il peut enfin participer aux audiences publiques afin de présenter son avis sur des sujets de sa compétence.

Il est trop tôt pour conclure au succès de ce comité. Cependant, si l'expérience de Kouchibouguac s'avère intéressante et positive, il est possible que Pares Canada invite d'autres parcs à constituer un tel comité

\section{Le cas du Parc national Kouchibouguac}

La collaboration avec le Parc national Kouchibouguac représente un modèle de collaboration intense et diversifiée. Les études et les stages qui y sont effectués ont des objectifs non seulement d'information sur les ressources du milieu, mais concernent aussi la conservation, l'aménagement, la gestion, le maintient de l'intégrité écologique ainsi quel'inventaire et l'évaluation d'impacts ou de risques d'impacts. Des étudiants de premier et de second cycle y sont impliqués. Les résultats des travaux sont avantageux pour les deux parties. En effet, d'une part, l'Universitéetsesétudiants en retirent des avantages scientifiques et académiquesalorsque, d'autre part, le Parc peut utiliser les résultats des recherches dans ses activités de planification et de gestion.

\section{Connaissance du milieu}

Le Parc national Kouchibouguac est situé sur la côte est du Nouveau-Brunswick, à la limite nord du détroit de Northumberland, soità une centaine de kilomètres au nord de Moncton. Il s'étend sur un territoire de $239 \mathrm{~km}^{2}$ dans le comté de Kent, entre Pointe-Sapin au nord et Richiboucto au sud. La partie littorale forme une baie limitée par des îles-barrières d'une longueur de $25 \mathrm{~km}$. La partie terrestre atteint 
d'est en ouest une largeur maximale de 13 $\mathrm{km}$. Ce territoire comprend huit grands types d'habitats (Desloges et coll., 1980): 1) la mer, 2) lecordonlittoral, 3) les estuaires et les lagunes, 4) les marais salés, 5) les habitats d'eau douce, 6) les tourbières, 7) les forêts et 8 ) les plaines.

Le nombre de touristes qui visitent le Parc national Kouchibouguac est en constante augmentation. Il se situait à 230000 personnes en 1994, soit une augmentation de $360 \%$ depuis 1981 . De ce nombre, entre 20000 et 30000 participent aux activités d'interprétation pour se familiariser avec les différents milieuxdu Parc, lesquels sont représentatifs de la côte est du NouveauBrunswick et même d'une partie de la côte est de l'Amérique du Nord où on retrouve unevaste plaine côtièreet un systèmed'ìlesbarrières. En effet, les motifs de visite du Parc sont en train de changer. En 1989, c'était le camping et la plage qui attiraient près de $60 \%$ des visiteurs alors que les autres activités, dont la randonnée à bicyclette, la contemplation du panorama et l'interprétation de la nature n'en attiraient que $40 \%$ (Service canadien des parcs, 1993). En 1994, les résultats d'une préenquête indiquaient que $65 \%$ des visiteurs venaient pour des vacances prolongées et surtout pour la beauté du site ( $25 \%$ ), la qualité du parc (23\%), la plage ( $23 \%$ ) ou par curiosité $(15 \%)$. De plus, le développement de la randonnée à bicyclette, beaucoup plus près de la nature, a supplanté le traditionnel tour d'auto pour contempler le panorama.

\section{Historique de la collaboration}

Amorcée en 1988 par un premier camp de travail éttudiant sur le terrain, la collaboration entre le Parc et le département de géographie et télédétection del'Université de Sherbrooke se poursuit encore aujourd'hui. Le Parc reçoit chaque année les étudiants quiyeffectuent la cueillette de l'information nécessaire à l'analyse d'une problématique liée à la connaissance du milieu et de la clientèle ou à la gestion des ressources.

Entre 1988 et 1994, plus de 200 étudiants ont ainsi produit 55 rapports que le parc utilise à divers niveaux pour ses activités de planification. De plus, les résultats des travaux et des discussions avec les gestionnaires du Parc ont permis de dégager des problématiques plus importantes qui ont rendu possible la réalisation de

\section{TABLEAU 1}

Mémoires de maitrise terminés ou en cours au Pare national Kouchibouguac

\begin{tabular}{|c|c|c|}
\hline Étudiant(e) & Thème de recherche & Années \\
\hline Denis Bernier & $\begin{array}{l}\text { Impacts environnementaux de l'épandage } \\
\text { du fénitrothion }\end{array}$ & $1989-1991$ \\
\hline Éric Tremblay & $\begin{array}{l}\text { Vers une gestion intégrée du dragage au } \\
\text { Parc national Kouchibouguac }\end{array}$ & $1989-1991$ \\
\hline Martin Lemire & Capacité de support pour les activités récréatives & 1991-1995 \\
\hline Brigitte Dagneau & Mode d'évolution du système d'iles-barrières & $1993-1995$ \\
\hline Sylvain Boucher & $\begin{array}{l}\text { Validation de la méthode d'identification des } \\
\text { colonies actives de castors par photo-interprétation }\end{array}$ & $1993-1995$ \\
\hline Patrick Dubois & Habitats pour l'implantation de colonies de castors & 1993-1995 \\
\hline Nancy Maillet & Evaluation de l'intégrité écologique & $1993-1995$ \\
\hline Martine Ruel & $\begin{array}{l}\text { Risque d'impact sur l'environnement dans } \\
\text { le grand ccosysteme }\end{array}$ & $1994-1996$ \\
\hline
\end{tabular}

\section{TABLFAU 2}

Priorités de gestion du Parc national Kouchibouguac et thèmes déjà étudiés par l'Université de Sherbrooke

Ressource

Moyen d'intervention

Gestion des écosystèmes

1-Banes de myes

2-Peche commerciale en estuaire

3-Activités illégales

C

4- Banque d'information du Parc

$\mathrm{C}$

5- Bar rayé

C, S, M

6- Iles-barrières

7-Salmonides

-..

8- Ecosystème régional du Parc

C, S, M

$\mathrm{C}$

9- Programme de monitoring de l'etat des Ecosystemes $\quad C, M$

10- Sternes et goélands

11- Amphibiens

12- Qualité de l'eau

$\mathrm{S}, \mathrm{M}$

$\mathrm{C}, \mathrm{M}$

$\cdots$

13- Polluants géostrophiques

M

14- Limites des changements acceptables

15- Faune regionale

16-Impacts de l'exploitation de la tourbe

17- Inventaire de la végétation $\quad M$

18- Inventaire des mammiferes: C, M

Ressources culturelles

1- Cimetières abandonnés

C

2- Erosion des sites archéologiques

C

$\mathrm{C}=$ rapport de camp de travail

$\mathrm{S}=$ stage cooperatif

M = mémoire de maitrise en géographie ou essai en environnement

Adapté de Tremblay et Beoch, 1994. 
deux essais à la maîttrise en environnement complétés en 1991, sous la direction d'un professeur de géographie, et de six mémoires de maîtrise en géographie terminés ou en cours de réalisation (tableau 2).

\section{Thèmes d'étude}

Le choix des sujets d'étude pour les camps de travail étudiants et les mémoires de maîtrise découleprincipalementdesthèmes retenus par le Plan de gestion du Parc (Service canadien des parcs, 1993). Ces derniers sont liésà deux objectifs généraux, soit la gestion du Parc ainsi que l'intérêt scientifique et académique des thèmes qui portent autant sur les écosystèmes que sur les activités récréatives.

Ainsi, au plan des écosystèmes, plusieurs études ont couvert ou vont couvrir en tout ou en partie les priorités de gestion des écosystèmes et des ressources culturelles définies dans le Plan de gestion par Tremblay et Beach (tableau 2).

D'autres études portent sur des thèmes d'activitésou d'infrastructures de récréation pour fins d'aménagement et pour évaluation des impacts. Plusieurs aménagements sont proposés comme des pistes cyclables, des pistes de ski de randonnée, l'aménagement de campings ou des agrandissements de campings classiques, des sites de camping primitifs, des aires de baignade, des aires de pique-nique, etc. En plus de ces dernières, d'autres études évaluent les impactssurl'environnement, la dégradation de campings primitifs, la sédimentation au voisinagedepasserelles flottantes, la teneur en cadmium des sols, les effets du piétinement dans les dunes, etc.

Enfin, plusieurs des thèmes d'étude retenus le sont pour des raisons d'ordre scientifique ou académique, soit la répartition des algues et des sédiments dans les lagunes, l'origine des sédiments de lagune déterminée par les isotopes du carbone, la classification des berges des cours d'eau et des rives des lagunes, la cartographie géomorphologique, la subsidence du littoral, etc.

\section{Conclusion}

Cette expérience soutenue de collaboration entre une université et un parc est enrichissante de part et d'autre tant au plan théorique que pratique, tant au plan éco- nomique que social. Elle mérite qu'on la prolonge en y consentant le temps et les ressources nécessaires.

Il faut aussi souligner quelques aspects que l'on a tendance à négliger, comme la découverte d'un milieu différent, le travail en équipe, le fonctionnement d'un parc national, la collaboration interuniversitaire entre les étudiants qui se manifeste par la discussion des sujets de recherche et l'entraide sur le terrain.

\section{Évaluation}

En 1991, Vallières évaluaitqualitativement les bénéfices et les problèmes reliés au partenariat entre Parcs Canada et les institutions d'enseignement. Quatreans plus tard, cetteévaluation est encorevalable, car le bilan demeure positif pour les deux parties.

En effet, il est toujours vrai que l'échange mutuel d'informations est enrichissant et quele fait de seréunir, d'échanger desidées et de travailler ensemble sur le terrain stimule la recherche et développe des idées nouvelles. Les étudiantes et les étudiants sont particulièrement gagnants car ils peuvent prendre une expérience pratique à partir de cas bien concrets.

Cette mise en commun des ressources est une excellente alternative à la décroissance généralisée des mesures de support à la recherchedepuis une décennie. Pour Parcs Canada, cela se traduit par une augmentation de la recherche dans ses parcs ainsi qu'une meilleure connaissance des ressources et des problèmes de conservation de ses pares à peu de frais. Une des conséquencesà long terme est la sensibilisation du public à la protection du patrimoine et la possibilité d'accélérer, pour compléter le réseau, la création de nouveaux parcs. Pour les institutions d'enseignement, cela se traduit par le développement d'une expertise dans les problèmes d'inventaire, d'interprétation et de conservation de la nature avec une certaine garantie de financerient, même si ce dernier est minime.

Dans l'avenir, l'accent devrait être mis sur le développement de projets reliés au suivi environnemental et au service d'interprétation, surtout en ce qui a trait au contenu et à la planification des programmes, ainsi qu'au service d'accueil. $f$

\section{RÉFE RENCES}

BERGERON, P. et FAUCHER, I. (1994) Sondages: Parc national Kouchibouguac, Département de géographie et télédétection, Unversitéde Sherbrooke, $10 \mathrm{p}$.

DESLOGES, C. et coll., Les ressources naturelles du pare national de Kouchibouguac, Parcs Canada, Région de l'Atlantique, 1980, $137 \mathrm{p}$.

ENVIAONNEMENT CANADA, L'etat desparcs; profils 1990, Service des pares, Ottawa, 1990a, 88 p.

ENVIAONNEMENT CANADA, L'état dos parcs: rapport 1990. Service des parcs, Ottawa, 1990b,229 p.

GOUVERNEMENT DU CANADA, Le plan vert du Canada, Approvisionnements et services Canada, Ottawa, 1990, $174 \mathrm{p}$.

LEMIRE. M. Évolution du concept de capacité de sup. port récrétif et application aLx activités de plein air, La géographie appliqube, vol. 10, no 3, 1992. pp. 6-10.

PATRIMOINE CANADIEN, Principes directeurs et politiques de gestion de Pares Canada, Approvisionnementset Services Canada, Ottawa, 1994. $127 \mathrm{p}$.

SERVICE CANADIEN DES PARCS, Manuel technique du programme de bunóvolat du Service des parcs, Environnement Canada, Ottawa, 1987, 94 $\mathrm{P}$

SERVICE CANADIEN DES PAFCS, Parc national Kouchibouguac: plan de gestlon, Enwironnement Canada, Ottawa, $1993,75 \mathrm{p}$.

TAEMBLAY, E. and $\mathrm{H}$. Beach, Ecosystem Conservation Plan, Kouchibouguac National Park, Canadian Parks Service, Kouchibouguac National Park, Natural Resource Conservation, 1994, 95 p.

VALLIERES, N. Canadien Parks Service Partnerships with Universities: A Preliminary Overview, Canadian Parks Service, Hull, 1991, 57 p.

WOODLEY, S. La surveillance de rintégrité des dcosystèmes dans les pares nationaux, Heritage Plesources Centre, University of Waterloo, 1991, $163 \mathrm{p}$. 